\section{IN BRIEF}

\section{$\Rightarrow$ SYSTEMIC LUPUS ERYTHEMATOSUS}

In-hospital SLE mortality has fallen and plateaued

In a nationwide analysis of hospitalizations in the USA between 2006 and $2016(n=340,467,049)$, the risk of death decreased from $2.2 \%$ to $1.5 \%$ during this period among patients with a diagnosis of systemic lupus erythematosus (SLE; $n=1,903,279$ ). Compared with non-SLE hospitalizations, SLE hospitalizations were more likely to end in death in 2006, but in-hospital survival for SLE hospitalizations improved from 2006 to 2008 and then plateaued, after which mortality was similar between the two groups. Mortality was highest among SLE populations classified as 'black', 'Hispanic' or 'Asian/Pacific Islander'.

ORIGINAL ARTICLE Anastasiou, C. et al. Mortality among Hospitalized Individuals with Systemic Lupus Erythematosus in the United States between 2006 and 2016. Arthritis Care Res. https://doi.org/10.1002/acr.24356 (2020)

\section{$\Rightarrow$ PAEDIATRIC RHEUMATOLOGY}

\section{Global prevalence of JIA, JSLE and club foot}

More than 3 million children or young adults worldwide are living with juvenile idiopathic arthritis (JIA), juvenile systemic lupus erythematosus (JSLE) or club foot, according to an analysis of the World Bank Group databank that included data from $>200$ countries. Of the 2 billion individuals aged $<16$ years who were included in the analysis, 2,069,246 had JIA and 206,931 had JSLE in 2017. Furthermore, an estimated 675,061 children aged $<5$ years had club foot. Asia had the highest prevalence of all three conditions, followed by Africa, the Americas, Europe and Oceania.

ORIGINAL ARTICLE Dave, M. et al. Global prevalence estimates of three chronic musculoskeletal conditions: club foot, juvenile idiopathic arthritis and juvenile systemic lupus erythematosus. Pediatr. Rheumatol. 18, 49 (2020)

\section{CLINICAL TRIALS}

\section{Long-term efficacy of canakinumab in crFMF}

In patients with colchicine-resistant familial Mediterranean fever (crFMF), open-label treatment with canakinumab (150 or $300 \mathrm{mg}$ every 4 or 8 weeks, with individually adjusted dose regimens) continued to control disease activity from week 40 in the phase III CLUSTER trial. During a 72-week period (weeks 41 to 113 of the trial), $>90 \%$ of the treated patients experienced no flares or had one flare, whereas a median of 17.5 flares per year was reported for the patients before entering the trial. The median C-reactive protein serum values remained low, and no new or unexpected adverse events were reported.

ORIGINAL ARTICLE Ozen, S. et al. Long-term efficacy and safety of canakinumab in patients with colchicine-resistant familial Mediterranean fever: results from the randomised phase III CLUSTER trial. Ann. Rheum. Dis. https://doi.org/10.1136/ annrheumdis-2020-217419 (2020)

\section{$\Rightarrow$ QUALITY OF LIFE}

\section{Increased risk of self-harm in fibromyalgia}

The risk of self-harm is increased in patients with fibromyalgia, osteoarthritis or rheumatoid arthritis, but not in patients with ankylosing spondylitis, compared with patients without these conditions, according to a retrospective cohort study. Analysis of data from the Clinical Practice Research Datalink found that between 1990 and 2016, the incidence of self-harm was highest in patients with fibromyalgia (25.12 (95\% Cl 22.45-28.11) per 10,000 person years), with these patients having a twofold increased risk of self-half compared with individuals without a rheumatic condition.

ORIGINAL ARTICLE Prior, J. A. et al. Rheumatological conditions as risk factors for self-harm: A retrospective cohort study. Arthritis Care Res. https://doi.org/10.1002/ acr.24345 (2020)

\title{
15000 \\ GENETICS \\ Complement: the missing genetic link for SLE and pSS?
}

Several rheumatic autoimmune diseases have strong genetic associations with the MHC locus; however, although definite links have been identified between these genetic associations and HLA molecules in rheumatoid arthritis, such clear links have not yet been identified for systemic lupus erythematosus (SLE) or primary Sjögren syndrome (pSS). The results of a study published in Nature suggest that the genetic association between the MHC locus and SLE and pSS could instead be due to the complement protein $\mathrm{C} 4$, which is encoded at the same locus.

$\mathrm{C} 4$ is encoded as two separate genes, $C 4 A$ and $C 4 B$, which are present at different copy numbers in different individuals. The copy numbers of $C 4$ genes can be imputed from combinations of specific single nucleotide polymorphisms, which enabled the authors of the new study to investigate the copy numbers of $C 4 A$ to $C 4 B$ in individuals with different diseases.

“Our lab's prior work on the association between copy number variation of the [C4 genes] and schizophrenia motivated us to see if this source of genetic variation could possibly explain other signals of common, heritable risk at the MHC locus of the human genome," explains corresponding author Nolan Kamitaki. "In particular, though previously attributed to various combinations of HLA class I and class II alleles, the associations for [SLE] and [pSS] risk in this region seemed to have a similar pattern of linkage disequilibrium as seen with $C 4$ variation itself."

Kamitaki and colleagues found that variation in the copy number of $C 4 A$ and $C 4 B$ was associated with a sevenfold difference in risk for SLE and a 16-fold difference in risk for pSS. For both diseases, an increased copy number of $C 4 A$ was strongly protective, and an increased copy number of $C 4 B$ was moderately protective. The researchers also investigated $H L A-D R B 1^{\star} 03: 01$, which had been thought to account for the association between the $C 4$ copy number and SLE, and found no consistent effect on SLE risk once $C 4$ variation was accounted for.

"Resolving the genetic association in [the MHC locus] to copy number variation of the $C 4$ gene[s] heavily suggests that the complement pathway is a fundamental contributor to the development of these autoimmune disorders rather than an observed outcome," states Kamitaki.

Interestingly, the protective effects of C4A in SLE and pSS are reversed in schizophrenia. As SLE and pSS are more common in women and schizophrenia is more common in men, the researchers investigated whether the effects of $C 4$ variation had a sex bias. They found that C4 genes are associated with larger effects in all three diseases in men than in women.

"Although the expression of $\mathrm{C} 4$ at the RNA level does not appear to differ between men and women, we saw that men had more $\mathrm{C} 4$ protein in both cerebrospinal fluid and blood plasma, suggesting that this [difference] may explain the greater genetic association in men," says Kamitaki. "This result suggests a molecular pathway contributing to the sex-bias in disease incidence".

Joanna Clarke

ORIGINAL ARTICLE Kamitaki, N. et al. Complement genes contribute sex-biased vulnerability in diverse disorders. Nature https:// doi.org/10.1038/s41586-020-2277-x (2020) 